\title{
The No Alternatives Argument
}

\author{
Richard Dawid* $\quad$ Stephan Hartmann $^{\dagger} \quad$ Jan Sprenger ${ }^{\ddagger}$
}

February 14, 2012

\begin{abstract}
Scientific theories are hard to find, and once scientists have found a theory $\mathrm{H}$, they often believe that there are not many distinct alternatives to $\mathrm{H}$. But is this belief justified? What should scientists believe about the number of alternatives to $\mathrm{H}$, and how should they change these beliefs in the light of new evidence? These are some of the questions that we will address in this paper. We also ask under which conditions failure to find an alternative to $\mathrm{H}$ confirms the theory in question. This kind of reasoning (which we call the No Alternatives Argument) is frequently used in science and therefore deserves a careful philosophical analysis.
\end{abstract}

\section{Introduction}

We typically confirm or disconfirm a scientific hypothesis with a piece of empirical evidence. For example, the observation of a black raven confirms the hypothesis that all ravens are black, and certain clicks in a particle detector confirm the existence of the top quark. However, there are situations where empirical evidence is unattainable over long periods of time. Such situations arise with particular force in contemporary high energy physics, where the characteristic empirical signatures of theories like Grand Unified Theories or string theory must be expected to lie many orders of magnitude beyond the reach of present day experimental technology. They are entirely common also in scientific fields such as palaeontology or anthropology, where scientists must rely on

\footnotetext{
* Department of Philosophy and Institute Vienna Circle, University of Vienna, Universitätsstr. 7, 1010 Vienna (Austria) - http://homepage.univie.ac.at/richard.dawid/ richard.dawid@univie.ac.at.

$\dagger$ Tilburg Center for Logic and Philosophy of Science, Tilburg University, PO Box 90153, 5000 LE, Tilburg (The Netherlands) - http://www.stephanhartmann.org s.hartmann@uvt.nl.

¥Tilburg Center for Logic and Philosophy of Science, Tilburg University, PO Box 90153, 5000 LE, Tilburg (The Netherlands) - http://www.laeuferpaar.de - j.sprenger@uvt.nl.
} 
the scarce and haphazard empirical evidence they happen to find in the ground. Interestingly, scientists are at times quite confident regarding the adequacy of their theories even when empirical evidence is largely or entirely absent. Trust in a theory $\mathrm{H}$ in such cases must be based on what we want to call non-empirical evidence for $\mathrm{H}$, that is, evidence that is neither deductively nor probabilistically implied by $\mathrm{H}$.

From an empiricist point of view, arguments relying on non-empirical evidence may be regarded as mere speculation: they netiher contribute to actual theory confirmation nor do they have objective scientific weight. We challenge this claim by focusing on the case where scientists develop a considerable degree of trust in a theory $\mathrm{H}$ based on the observation that no alternatives to $\mathrm{H}$ have been found, despite considerable efforts to do so. We call this argument the No Alternatives Argument (NAA).

In order to formalize this argument, we introduce the concept of the number of alternative theories to $\mathrm{H}$ (Sect. 2) and study how our beliefs about the number of alternatives respond to empirical evidence (Sect. 3). On that basis, we construct a probabilistic model of NAA and prove the possibility of non-empirical theory confirmation (Sect. 4). Next, we show that the significance of NAA in scientific reasoning depends on the scientists' subjective judgments. An agreement on these judgments might be achieved by what we call the Meta-Inductive Argument (MIA), which we tentatively explore in Sect. 5. Finally, we put our findings into a broader context and briefly look at applications in epistemology and philosophy of science (Sect. 6). Throughout this paper, we operate in the framework of Bayesian epistemology. ${ }^{1}$

\section{The conceptual framework}

In order to understand the problem of non-empirical theory confirmation, we contrast it with its empirical counterpart. We call some evidence E empirical evidence for $\mathrm{H}$ if and only if (i) $\mathrm{H}$ predicts $\mathrm{E}$ and (ii) $\mathrm{E}$ is observed. The evidence E can be observed perceptually or by means of measurement instruments, as common in modern science. If $\mathrm{T}$ denotes the statement that hypothesis $\mathrm{H}$ is empirically adequate, then this amounts to $P(\mathrm{E} \mid \mathrm{T})>P(\mathrm{E})$, or in a more familiar form, $P(\mathrm{~T} \mid \mathrm{E})>P(\mathrm{~T})$. Bayesian epistemologists use this inequality as a criterion for whether $\mathrm{E}$ confirms $\mathrm{T}$.

\footnotetext{
${ }^{1}$ Recent surveys of Bayesian epistemology are Hájek and Hartmann (2010) and Hartmann and Sprenger (2010). Applications of Bayesian epistemology to scientific reasoning are given in Howson and Urbach (2006).
} 
Non-empirical evidence $\mathrm{F}$ for a theory $\mathrm{H}$ is evidence that is neither deductively nor probabilistically implied by $\mathrm{H}$. In other words, F exemplifies evidence that does not fall into the intended domain of $\mathrm{H}$ or a related scientific theory. Then, how is it possible at all that $\mathrm{F}$ is evidence for H? Does $\mathrm{F}$ qualify as evidence in an argument from ignorance (Walton 1995, Hahn and Oaksford 2007, Sober 2009), such as: if $\mathrm{H}$ were not empirically adequate, then we would have disproved it?

The most plausible way to solve this problem is to deploy a two step process. First, we find a statement that does predict evidence of the type F. Then, we show that this statement is probabilistically relevant to the empirical adequacy of $\mathrm{H}$. In the case of NAA, our non-empirical evidence $\mathrm{F}_{\mathrm{A}}$ consists in the fact that scientists have not found any alternatives to a specific solution of a research problem, despite looking for them with considerable energy and for a long time. Then it is straightforward to identify a natural candidate for a statement that predicts $\mathrm{F}_{\mathrm{A}}$, namely that the number of alternative theories to $\mathrm{H}$ is small. If there were only very few alternatives to $\mathrm{H}$, then this would render $\mathrm{F}_{\mathrm{A}}$ more likely than a scenario where a huge number of possible alternative theories can be constructed: in the latter case, one might expect that scientists would have found one of them already.

The number $k$ of possible scientific theories which can account for a certain set of data is in turn relevant for the probability of the empirical adequacy of H. We assume that scientists who develop a theory in accordance with available data do not have a perfectly reliable method to select the true theory if false theories can be constructed which also reproduce the available data. This assumption seems to be fairly plausible in science: scientists often come up with an incorrect, but fruitful theory when they begin to investigate a new field. Bohr's model of the atom is a good example for this claim.

Based on the above reasoning, we introduce a random variable $Y$ measuring the number of alternatives to $\mathrm{H}$, and the set of propositions $\mathrm{Y}_{\mathrm{k}}:=\{Y=k\}$ expressing that there are $k$ adequate and distinct alternatives which can account for the available data $\mathrm{E}$. We will later show that, via its effect on the $\mathrm{Y}_{\mathrm{k}}$, the non-empirical evidence $\mathrm{F}_{\mathrm{A}}$ confirms $\mathrm{H}$ under plausible conditions. ${ }^{2}$

Note that any inference about the number of alternatives to a theory $\mathrm{H}$ requires an account of what counts as an alternative to a given theory and how scientific theories are individuated. Such an account will depend on the specific

\footnotetext{
${ }^{2}$ Throughout this paper we follow the convention that propositional variables are printed in italic script, and that the instantiations of these variables are printed in roman script. See Bovens and Hartmann (2003).
} 
scientific context, and scientists typically have a good grip on what counts as a distinct theory. There are, however, two conditions that are worth stressing and that are important for the following discussion.

First, different theories make different predictions. If two theories make exactly the same predictions, then we consider them to be identical. For example, we consider the De Broglie-Bohm version and the Copenhagen version of quantum mechanics as representing the same theory (Cushing 1994). As a consequence, we are only interested in arriving at empirically adequate theories, and not in the more ambitious goal of finding true theories (cf. van Fraassen 1980).

Second, different theories provide different solutions to a given scientific problem. That is, theories which only differ in a detail, say in the value of a parameter, or the existence of a physically meaningless dummy variable, do not count as different theories. For example, the Higgs model in particle physics is treated as one theory, although the hypothesized (and perhaps finally discovered) Higgs particle could have different mass values. What is at stake here is the general adequacy of the Higgs model as a theoretical mechanism that can explain particle masses with the help of a scalar field.

This condition makes it plausible that the number of alternatives to a given theory is finite. If it were enough to slightly modify the value of a certain parameter in order to arrive at a new theory, then coming up with new theories would be an easy and not very creative task. However, inventing a novel mechanism, or telling a new story of why a certain phenomenon came about is much harder. It is not so plausible that there is an infinite number of such distinct stories. This brings us to the next question: what can empirical evidence tell us about the (probable) number of alternatives to a given theory $\mathrm{H}$ ?

\section{Assessing the number of alternative theories}

Let us assume that an agent is convinced that the number of alternatives $Y$ to a theory $\mathrm{H}$ is finite. Then a particularly interesting belief structure may arise, namely when she also asserts that the expected number of alternatives to $H$ is infinite. Formally, we can express this tension as follows (proof in appendix A):

Proposition 1. For any $N \in \mathbb{N}$ and any $\varepsilon>0$, an agent's belief function $P$ may jointly satisfy (i) $P(Y=\infty)=0$, (ii) $P(Y \leq N) \geq 1-\varepsilon$, and (iii) $\langle Y\rangle=\sum_{k=0}^{\infty} k P\left(\mathrm{Y}_{\mathrm{k}}\right)=\infty$.

In this notation, $\langle Y\rangle$ denotes the expectation value of $Y$. In other words, an agent might rule out an infinite number of alternatives to $\mathrm{H}$, be strongly con- 
vinced that there are few alternatives to $\mathrm{H}$, and yet retain the belief that our best guess regarding the number of alternatives to $\mathrm{H}$ is "infinitely many" or "greater than any number that we can imagine". This phenomenon is well-known from paradoxes of decision theory, such as the valuation of the St. Petersburg Game, but to our knowledge, its epistemic counterparts have not been explored before.

In other words, Proposition 1 points out the possibility of a strong epistemic tension within a single agent regarding the number of alternatives to a theory $\mathrm{H}$. This tension transfers to the agent's assessment of the problem of theoretical underdetermination: she might believe that $\mathrm{H}$ is fundamentally underdetermined by evidence (because our best guess for the number of alternatives is infinity), but at the same time be strongly convinced that elimination of a small set of alternatives eventually leads us to the empirically adequate theory.

Let us now study whether such a belief structure is responsive to evidence $\mathrm{E}$, be it empirical or non-empirical. First, we ask under which circumstances evidence $\mathrm{E}$ lowers the expected number of alternatives. In answer to this question, we can demonstrate the following theorem (proof in appendix A):

Theorem 1. Let $\mathrm{Y}_{\mathrm{k}}^{+}$denote the proposition that there are at least $k$ alternatives to theory $H$, and let $\mathrm{Y}_{\mathrm{k}}^{-}$denote the proposition that there are at most $k-1$ alternatives to $H$. Then, if $P\left(\mathrm{E} \mid \mathrm{Y}_{\mathrm{k}}^{+}\right) \leq P\left(\mathrm{E} \mid \mathrm{Y}_{\mathrm{k}}^{-}\right)$for all $k \in \mathbb{N}$ and $P\left(\mathrm{E} \mid \mathrm{Y}_{\mathrm{k}}^{+}\right)<$ $P\left(\mathrm{E} \mid \mathrm{Y}_{\mathrm{k}}^{-}\right)$for at least one $k>0$, it will also be the case that $\langle Y\rangle>\langle Y\rangle_{\mathrm{E}}$, the latter expression denoting the expectation value of $Y$ under $P(\cdot \mid \mathrm{E})$.

In other words, if evidence $\mathrm{E}$ is more likely given a small rather than a large number of alternatives to $\mathrm{H}$, then the expected number of alternatives will be smaller a posteriori than it was a priori.

The condition of the theorem can be satisfied by empirical as well as nonempirical evidence. For non-empirical evidence such as $\mathrm{F}_{\mathrm{A}}:=$ "the scientists have not yet found an alternative to $\mathrm{H}^{\prime}$, it is easy to see that this evidence is the more likely the less alternatives there are. (See condition A3 in the next section.) Also, the required condition seems very plausible with respect to contrastive empirical evidence $\mathrm{E}$ predicted by theory $\mathrm{H}$. The more alternative theories exist, the less likely it is that the observed data are correctly predicted by $\mathrm{H}$, but not by its competitors.

Second, we ask the following question: Can an agent who believes that $\langle Y\rangle=\infty$ come to the belief that $\langle Y\rangle_{\mathrm{E}}<\infty$ ? Indeed, she can. The following theorem characterizes that case by stating four different sufficient conditions for such a belief change (proof in appendix A).

Theorem 2. Assume that $\langle Y\rangle=\infty$. Then any of the following conditions on evidence $E$ with $P(\mathrm{E}) \neq 0$ is sufficient for $\langle Y\rangle_{\mathrm{E}}<\infty$. 
1. The sequence $\left(k \cdot P\left(\mathrm{E} \mid \mathrm{Y}_{\mathrm{k}}\right)\right)_{\mathrm{k} \in \mathbb{N}}$ is bounded.

2. There are $\alpha, \beta>0$ be such that $\alpha+\beta>2$, and that $\left(k^{\alpha} P\left(\mathrm{E} \mid \mathrm{Y}_{\mathrm{k}}\right)\right)_{\mathrm{k} \in \mathbb{N}}$ and $\left(k^{\beta} P\left(\mathrm{Y}_{\mathrm{k}}\right)\right)_{\mathbf{k} \in \mathbb{N}}$ are bounded.

3. $\sum_{k=0}^{\infty} P\left(\mathrm{E} \mid \mathrm{Y}_{\mathrm{k}}\right)<\infty$ and there is a $N_{0} \in \mathbb{N}$ such that $\left(P\left(\mathrm{Y}_{\mathrm{k}}\right)\right)_{\mathrm{k} \in \mathbb{N}}$ is, for all $k \geq N_{0}$, monotonically decreasing.

4. $P\left(\mathrm{E} \mid \mathrm{Y}_{\mathrm{k}}\right) \rightarrow 0$ and there is an $\alpha>0$ such that

$$
\limsup _{k \rightarrow \infty} k^{2+\alpha}\left|P\left(\mathrm{E} \mid \mathrm{Y}_{\mathrm{k}}\right)-P\left(\mathrm{E} \mid \mathrm{Y}_{\mathrm{k}-1}\right)\right|<\infty .
$$

These four conditions have different rationales, but all of them constrain the rate of decline of $P\left(\mathrm{E} \mid \mathrm{Y}_{\mathrm{k}}\right)$ as $k$ increases. That is, the more alternatives there are, the less likely is $\mathrm{E}$. The first and second condition could also be expressed as $P\left(\mathrm{E} \mid \mathrm{Y}_{\mathrm{k}}\right) \in \mathcal{O}\left(1 / k^{\alpha}\right)$ for a suitable exponent $\alpha>0$. The third condition makes a similar constraint by demanding that $\sum_{k=0}^{\infty} P\left(\mathrm{E} \mid \mathrm{Y}_{\mathrm{k}}\right)$ converges, and the fourth condition controls the differences between the values of $P\left(\mathrm{E} \mid \mathrm{Y}_{\mathrm{k}}\right)$ for neighboring values of $k$.

Note that only the second condition makes an assumption about the rate of decline of $P\left(\mathrm{Y}_{\mathrm{k}}\right)$. This is in line with the idea that we have little grip on the rational beliefs about the number of empirically adequate alternatives, whereas we are in a better position to assess how our evidence $\mathrm{E}$ is affected by the number of alternatives.

As already stated, the punch line of all four conditions is that $P\left(\mathrm{E} \mid \mathrm{Y}_{\mathrm{k}}\right)$ converges fast enough to zero. For evidence $\mathrm{E}$ that is related to an empirical test of $\mathrm{H}$, this assumption is reasonable: if there are more and more alternatives, why should $\mathrm{H}$, instead of an unconceived alternative (Stanford 2006), survive empirical tests? Thus, if large values of $Y$ make little difference regarding our trust in the predictions of $\mathrm{H}$, then we will abandon the belief that the expected number of alternatives is infinite. This is exactly what we would expect intuitively.

Conversely, we may also ask the question: can an agent who believes that $Y$ takes finite values only (i.e., that $\langle Y\rangle\left\langle\infty\right.$ ) come to the belief that $\langle Y\rangle_{\mathrm{E}}=\infty$ for some evidence $\mathrm{E}$ ? The answer to this question is a no. No empirical evidence is able to overturn the verdict that the expected number of alternatives to $\mathrm{H}$ is finite (proof in appendix A):

Proposition 2. If $\langle Y\rangle<\infty$, then for any evidence $E$ (empirical or nonempirical) with $P(\mathrm{E}) \neq 0,\langle Y\rangle_{\mathrm{E}}<\infty$.

This means that the belief that the expected number of alternatives is finite is not responsive to empirical evidence: once you believe it, you will always 
believe it, independently of which evidence you receive. This points to an interesting asymmetry: evidence can change the belief that there are infinitely many alternatives, but it cannot change the belief that there are finitely many alternatives. The asymmetry between Theorem 2 and Proposition 2 confirms a suspicion that Theorem 1 has already prompted, namely that empirical evidence usually lowers the expected number of alternatives. This finding might help to explain the convergence (rather than divergence) of scientific inquiry.

In the following section, we investigate whether non-empirical evidence can lower the expected number of alternatives and indirectly confirm the currently best theory.

\section{The No Alternatives Argument}

Having investigated the belief dynamics for the number of alternatives to a theory $\mathrm{H}$, we now proceed to a formal analysis of the No Alternatives Argument (NAA). In this case, the non-empirical evidence consists in the observation that scientists have not yet found an alternative to $\mathrm{H}$. In accordance with our previous analysis, this observation is taken to indicate that, in some sense, there are actually not too many alternatives to H. Focusing on the case of string theory, Dawid $(2006,2009)$ calls this the argument of no choice.

Following this line of reasoning, we will reconstruct NAA based on the notion that there exists a specific but unknown number $k$ of possible scientific theories. These theories have to be compatible with a set of constraints $\mathcal{C}$ - whose nature is left to the scientific community, cf. Sect. $2-$, to be consistent with the existing data $\mathcal{D}$, and to give distinguishable predictions for the outcome of some set $\mathcal{E}$ of future experiments. We will then show that failure to find an alternative to $\mathrm{H}$ raises the probability of $\mathrm{H}$ being empirically adequate and thus confirms $\mathrm{H}$.

To do so, we introduce the binary propositional variables $T$ and $F_{A}$, already briefly encountered in Sect. 2. $T$ has the values: $\mathrm{T}$ : "The hypothesis $\mathrm{H}$ is empirically adequate", and $\neg \mathrm{T}$ : "The hypothesis $\mathrm{H}$ is not empirically adequate". The propositional variable $F_{A}$ has the values: $\mathrm{F}_{\mathrm{A}}$ : "The scientific community has not yet found an alternative to $\mathrm{H}$ that fulfills $\mathcal{C}$, explains $\mathcal{D}$ and predicts the outcomes of $\mathcal{E}$ ", and $\neg \mathrm{F}_{\mathrm{A}}$ : "The scientific community has found an alternative to $\mathrm{H}$ that fulfills $\mathcal{C}$, explains $\mathcal{D}$ and predicts the outcomes of $\mathcal{E}$ ".

We would like to explore under which conditions $\mathrm{F}_{\mathrm{A}}$ confirms $\mathrm{H}$, that is, when

$$
P\left(\mathrm{~T} \mid \mathrm{F}_{\mathrm{A}}\right)>P(\mathrm{~T}) .
$$

This equation suggests a direct influence of $T$ on $F_{A}$. See Figure 1 for a Bayesian 


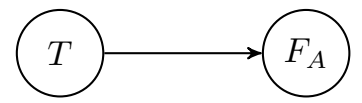

Figure 1: The Bayesian Network representation of the two-propositions scenario.

Network representation of this scenario. But since such a direct influence is blocked by the non-empirical nature of $F_{A}$, we introduce a third variable $Y$ which mediates the connection between $T$ and $F_{A}$. Like in the previous section, $Y$ has values in the natural numbers, and $\mathrm{Y}_{k}$ corresponds to the proposition that there are exactly $k$ hypotheses that fulfill $\mathcal{C}$, explain $\mathcal{D}$ and predict the outcomes of $\mathcal{E}$.

We should also note that the value of $F_{A}$ - that scientists find/do not find an alternative to $\mathrm{H}$ - does not only depend on the number of available alternatives, but also on the the complexity of the problem, the cleverness of the scientists, or the available computational, experimental, and mathematical resources. Call the variable that models the difficulty of the problem $D$, and let it take values in the natural numbers, with $\mathrm{D}_{\mathrm{j}}:=\{D=\mathrm{j}\}$ and $d_{j}:=P\left(\mathrm{D}_{\mathrm{j}}\right)$. The higher the values of $D$, the more difficult the problem. It is clear that $D$ has no direct influence on $Y$ and $T$ (or vice versa), but that it matters for $F_{A}$ and that this influence has to be represented in our Bayesian Network.

We now list five plausible assumptions that we need for showing the validity of the No Alternatives Argument.

A1. The variable $T$ is conditionally independent of $F_{A}$ given $Y$ :

$$
T \Perp F_{A} \mid Y
$$

Hence, learning that the scientific community has not yet found an alternative to $\mathrm{H}$ does not alter our belief in the empirical adequacy of $\mathrm{H}$ if we already know that there are exactly $k$ viable alternatives to $\mathrm{H}$.

This is our the most important assumption, and we consider it to be eminently sensible. Figure 2 shows the corresponding Bayesian Network. To complete it, we have to specify the prior distribution over $Y$ and the conditional distributions over $F_{A}$ and $T$, given the values of their parents. This is done in the following four assumptions.

A2. The prior probabilities

$$
y_{k}:=P\left(\mathrm{Y}_{\mathrm{k}}\right)
$$

are smaller than 1 , that is, $0 \leq y_{k}<1$. 


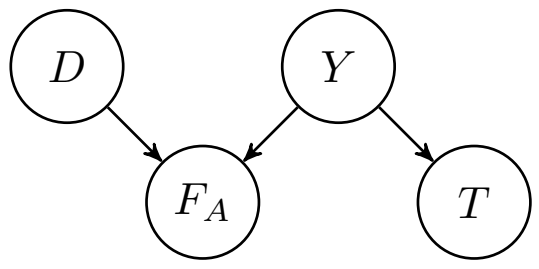

Figure 2: The Bayesian Network representation of the four-propositions scenario.

This assumption reflects the fact that we do not know the number of viable alternatives a priori.

A3. The conditional probabilities

$$
f_{k j}:=P\left(\mathrm{~F}_{\mathrm{A}} \mid \mathrm{Y}_{\mathrm{k}}, \mathrm{D}_{\mathrm{j}}\right)
$$

are monotonically decreasing in $k$ for all $j \in \mathbb{N}$ and monotonically increasing in $j$ for all $k \in \mathbb{N}$.

The decrease in the first argument reflects the intuition that the more alternative theories there are, the more likely it is that the scientists find at least one of them, given a certain level of difficulty. The increase in the second argument reflects the intuition that the more difficult a problem gets, the less likely it is that scientists find an alternative to $\mathrm{H}$, provided that the number of alternatives to $\mathrm{H}$ is fixed.

A4. The conditional probabilities

$$
t_{k}:=P\left(\mathrm{~T} \mid \mathrm{Y}_{\mathrm{k}}\right)
$$

are monotonically decreasing in $k$.

This assumption reflects the intuition that the more alternative theories there are, the less likely it is that scientists have identified the right one.

A5. There is at least one pair $(i, k)$ with $i<k$ for which (i) $y_{i} y_{k}>0$, (ii) $f_{i j}>f_{k j}$ for some $j \in \mathbb{N}$, and (iii) $t_{i}>t_{k}$.

Note that this assumption follows from A2, A3 and A4 if we replace "monotonically decreasing" by "strictly monotonically decreasing" in $\mathbf{A} 3$ and A4. However, to prove the following theorem, the weaker formulation suffices.

With these five assumptions, we can show that (proof in appendix B): 
Theorem 3. If assumptions $\mathbf{A} \mathbf{1}$ to $\mathbf{A} 5$ hold, then $\mathrm{F}_{\mathrm{A}}$ confirms $\mathrm{T}$, that is, $P\left(\mathrm{~T} \mid \mathrm{F}_{\mathrm{A}}\right)>P(\mathrm{~T})$.

We have therefore shown that $\mathrm{F}_{\mathrm{A}}$ confirms the empirical adequacy of $\mathrm{H}$ under rather weak and plausible assumptions. Note that $\mathrm{F}_{\mathrm{A}}$ does not confirm $\mathrm{T}$ if we believe, for example, with probability one that the number of alternatives has a certain value (e.g., infinity). Moreover, the degree of confirmation depends on the specific values of the parameters that occur in A2-A5 (for details, see appendix B).

\section{The significance of NAA}

We have seen that NAA can be used in support of a proposed theory. The question remains, however, whether the resulting support is of significant strength and whether using NAA in a specific situation is justified.

The Bayesian Network representation of NAA in Figure 2 suggests that such significance is difficult to attain by NAA on its own without further supportive reasoning. According to Figure 2, $\mathrm{F}_{\mathrm{A}}$ may confirm an instance of $D$ (limitations to the scientists' abilities to solve difficult problems) as well as an instance of $Y$ (limitations to the number of possible theories). From the Bayesian Network depicted in Figure 2, it is easy to see that for all $l \in \mathbb{N}$,

$$
P\left(\mathrm{D}_{\mathrm{l}} \mid \mathrm{F}_{\mathrm{A}}\right)=\frac{P\left(\mathrm{D}_{\mathrm{l}}, \mathrm{F}_{\mathrm{A}}\right)}{P\left(\mathrm{~F}_{\mathrm{A}}\right)}=\frac{d_{l} \cdot \sum_{k} y_{k} f_{k l}}{\sum_{j, k} d_{j} y_{k} f_{k j}} .
$$

Hence the ratio measure of confirmation is given by

$$
r\left(\mathrm{D}_{\mathrm{l}}, \mathrm{F}_{\mathrm{A}}\right):=\frac{P\left(\mathrm{D}_{1} \mid \mathrm{F}_{\mathrm{A}}\right)}{P\left(\mathrm{D}_{\mathrm{l}}\right)}=\frac{\sum_{k} y_{k} f_{k l}}{\sum_{j, k} d_{j} y_{k} f_{k j}} .
$$

We cannot provide fully general conditions for when this expression is greater than 1. However, we observe that the expression on the right hand side of equation (8) is monotonically increasing in $l$ since the $f_{k l}$ are monotonically increasing in $l$ for fixed $k$ (see assumption A3). That is, the degree of confirmation that $\mathrm{F}_{\mathrm{A}}$ lends to $\mathrm{D}_{l}$, as expressed by the ratio measure, increases with $l$. Thus, $\mathrm{F}_{\mathrm{A}}$ typically confirms the claim that the problem at hand is rather complicated (i.e., that it has a high $\operatorname{rank} l$ ) and typically disconfirms the claim that it is not particularly complicated (i.e., that it has a low rank $l$ ). The turning point $l^{*}$ depends on the precise values of the parameters in question.

To accentuate the resulting problem, note that the situation could be such that $\mathrm{D}^{*}:=\left\{D \geq l^{*}\right\}$ - the proposition that the problem has difficulty rank $l^{*}$ or higher - receives more confirmation than $\mathrm{T}$. While failure to find an alternative 
confirms the empirical adequacy of $\mathrm{H}$, this failure would also confirm, and to a larger degree, the hypothesis that the problem is too complicated for our current science. This alternative explanation of $\mathrm{F}_{\mathrm{A}}$ weakens the significance of NAA. To successfully apply NAA, one has to show that $\mathrm{F}_{\mathrm{A}}$ confirms $\mathrm{T}$ more than $\mathrm{D}^{*}$, but such a claim is sensitive to the specific parameter assignments and therefore hard to prove in general.

So far we have left the parameters $d_{k}, f_{k j}, t_{k}$ and $y_{k}$ largely unrestricted and assumed that they reflect the subjective degrees of belief of a scientist. Hence, different scientists may assign different values to these parameters, which implies that the significance of NAA will differ from scientist to scientist. In the absence of any further rational constraints, different scientists may come to radically different conclusions. Given that science aspires for objectivity, this is an unfortunate situation. In the remainder of this section, we sketch a reasoning procedure, called the Meta-Inductive Argument (MIA), that ensures agreement on the prior probabilities $y_{k}$, that is, on the distribution of the number of alternative theories.

The gist of MIA is best illustrated by a special case. It is notoriously difficult to find a theory that makes the correct predictions, rather than just accommodating existing data (Kahn et al. 1992, Hitchcock and Sober 1994). But remarkably, scientists have often succeeded at identifying that theory. Now, if there are a lot of alternative solutions to a given problem, then there is no reason to assume that the scientists identified the one theory which will prevail in the future. Thus, repeated predictive success within a particular scientific research program seems to justify the assumption that there may be few alternative theories in the given theoretical context.

Now, assume that a novel theory $\mathrm{H}$ shows similarities to theories $\mathrm{H}_{1}, \mathrm{H}_{2}$, etc., in the same scientific research program. The joint feature of these theories may be a certain theoretical approach, a shared assumption, or any other relevant characteristic. Let us assume that a substantial share of the theories to which $\mathrm{H}$ is similar have been empirically confirmed. Assume further that for those theories, we have empirically grounded posterior beliefs about the number of alternatives. Then, it seems reasonable to use these posteriors as priors for the number of alternatives to $\mathrm{H}$. After all, $\mathrm{H}$ is quite similar to $\mathrm{H}_{1}, \mathrm{H}_{2}$, etc. Statisticians routinely use this way of determining "objective" prior beliefs and refer to it as the empirical Bayes method (Carlin and Louis 2000).

If this move is accepted, then one is in a much better position to appreciate the significance of NAA, due to agreement on the prior probabilities of the $\mathrm{Y}_{\mathrm{k}}$. Admittedly, this account of MIA remains informal and provides at best a 
partial justification for the practical significance of NAA. On the other hand, formalizing MIA and strengthening the link between both arguments strikes us as a promising route for further research.

\section{Conclusions}

In this paper, we have completed three tasks: (i) we have studied the problem of theoretical underdetermination from the angle of how beliefs about the number of alternatives to a theory change in the light of evidence; (ii) we have formalized the No Alternatives Argument and explored under which conditions non-empirical evidence confirms a scientific theory $\mathrm{H}$; and (iii) we have sketched the Meta-Inductive Argument that allows us to assess the number of alternatives to $\mathrm{H}$ before empirical evidence in favor or against $\mathrm{H}$ is found.

In future work, we plan to relate the formal account given in this paper more closely to case studies from science. Here we are particularly interested in the case of string theory and the reasoning strategies employed in fields such as palaeontology and anthropology where contingent evolutionary details have to be reconstructed based on scarce and highly incomplete evidence. We will explore what role NAA plays in these fields, and how good the argument actually is.

There are also two philosophical applications which we would like to point out. First, Inference to the Best Explanation (Douven 2011, Lipton 2004) can, to a certain extent, be explicated in terms of NAA. In as much as the notion "best explanation" is understood as "the only genuinely satisfactory explanation", the fact that no other genuinely satisfactory explanation has been found can play the role of the claim of no alternatives in our argument, supporting the empirical adequacy of the currently best explanation.

Second, one may ask whether NAA could also play a role in confirming general philosophical theories. The reputation of a philosophical theory is often based on the understanding that no other consistent answer has been found or is perhaps not even conceivable. Can reasoning of this kind be supported by NAA? In principle, the answer to this question is yes, but there is a problem: philosophical theories do not have a record of empirical testing. Thus, we will be unable to quantify the significance of NAA with empirical data. Philosophy thus provides us with a neat example of the promises and limits of non-empirical theory confirmation beyond scientific contexts. 


\section{A Proof of the results in Section 3}

Proof of Proposition 1: The proof proceeds by construction. For instance, let $P(Y \leq N)=1-\varepsilon$, let $P\left(\mathrm{Y}_{\mathrm{k}}\right)=\mathrm{C} / \mathrm{k}^{2} \forall \mathrm{k}>\mathrm{N}$, and choose $C$ such that $\sum_{k>N} P\left(\mathrm{Y}_{\mathrm{k}}\right)=\varepsilon$ is satisfied. (The series $\sum_{k} 1 / k^{2}$ converges.) Then, it is easy to check that

$$
\langle Y\rangle \geq \sum_{k=N+1}^{\infty} k P\left(\mathrm{Y}_{\mathrm{k}}\right) \geq C \sum_{k=N+1}^{\infty} \frac{1}{k}=\infty
$$

Proof of Theorem 1: Let us define

$$
\mathrm{Y}_{\mathrm{k}}^{+}:=\{Y \geq k\} \quad \mathrm{Y}_{\mathrm{k}}^{-}:=\{Y<k\}
$$

We have assumed that $P\left(\mathrm{E} \mid \mathrm{Y}_{\mathrm{k}}^{+}\right) \leq P\left(\mathrm{E} \mid \mathrm{Y}_{\mathrm{k}}^{-}\right) \forall k \in \mathbb{N}$, with inequality for at least one $k>0$. Since $\mathrm{Y}_{\mathrm{k}}^{+}$and $\mathrm{Y}_{\mathrm{k}}^{-}$are an exhaustive partition of the probability space, this entails that $Y_{k}^{+}$and $E$ are negatively relevant to each other, and that

$$
P\left(\mathrm{Y}_{\mathrm{k}}^{+} \mid \mathrm{E}\right) \leq P\left(\mathrm{Y}_{\mathrm{k}}^{+}\right) \forall k \in \mathbb{N},
$$

with inequality for at least one $k>0$. Since $P\left(\mathrm{Y}_{\mathrm{k}}\right)=P\left(\mathrm{Y}_{\mathrm{k}}^{+}\right)-P\left(\mathrm{Y}_{\mathrm{k}+1}^{+}\right)$, we obtain by a simple diagonalization trick

$$
\begin{aligned}
\langle Y\rangle & =\sum_{k=0}^{\infty} k P\left(\mathrm{Y}_{\mathrm{k}}\right) \\
& =\sum_{k=0}^{\infty}\left(k P\left(\mathrm{Y}_{\mathrm{k}}^{+}\right)-k P\left(\mathrm{Y}_{\mathrm{k}+1}^{+}\right)\right) \\
& =0 \cdot P\left(\mathrm{Y}_{0}^{+}\right)+\sum_{k=1}^{\infty}\left(k P\left(\mathrm{Y}_{\mathrm{k}}^{+}\right)-(k-1) P\left(\mathrm{Y}_{\mathrm{k}}^{+}\right)\right) \\
& =\sum_{k=1}^{\infty} P\left(\mathrm{Y}_{\mathrm{k}}^{+}\right),
\end{aligned}
$$

and similarly

$$
\langle Y\rangle_{\mathrm{E}}=\sum_{k=1}^{\infty} P\left(\mathrm{Y}_{\mathrm{k}}^{+} \mid \mathrm{E}\right)
$$

Combining (10) and (11), we conclude

$$
\langle Y\rangle_{\mathrm{E}}=\sum_{k=1}^{\infty} P\left(\mathrm{Y}_{\mathrm{k}}^{+} \mid \mathrm{E}\right)<\sum_{k=1}^{\infty} P\left(\mathrm{Y}_{\mathrm{k}}^{+}\right)=\langle Y\rangle
$$

because of $P\left(\mathrm{Y}_{\mathrm{k}}^{+} \mid \mathrm{E}\right) \leq P\left(\mathrm{Y}_{\mathrm{k}}^{+}\right) \forall k \in \mathbb{N}$ (see (9)), and because we have assumed inequality for at least one $k>0$. 
Proof of Theorem 2: Proof of the first statement. Assume that the expression $\left(k \cdot P\left(\mathrm{E} \mid \mathrm{Y}_{\mathrm{k}}\right)\right)_{k \in \mathbb{N}}$ is bounded, that is, there is a $B>0$ such that $k \cdot P\left(\mathrm{E} \mid \mathrm{Y}_{\mathrm{k}}\right)<B$. Then it will be the case that

$$
\begin{aligned}
\langle\mathrm{Y}\rangle_{\mathrm{E}} & =\frac{1}{P(\mathrm{E})} \sum_{k=1}^{\infty} k P\left(\mathrm{Y}_{\mathrm{k}}\right) P\left(\mathrm{E} \mid \mathrm{Y}_{\mathrm{k}}\right) \\
& \leq B \cdot \frac{1}{P(\mathrm{E})} \sum_{k=1}^{\infty} P\left(\mathrm{Y}_{\mathrm{k}}\right) \\
& <\infty
\end{aligned}
$$

proving the sufficiency of the first condition.

Related to this is the case that $k^{\alpha} \cdot P\left(\mathrm{E} \mid \mathrm{Y}_{\mathrm{k}}\right) \leq \mathrm{A}_{\alpha}$ and $k^{\beta} \cdot P\left(\mathrm{Y}_{\mathrm{k}}\right) \leq \mathrm{A}_{\beta}$ for all $k \in \mathbb{N}$ and some constants $A_{\alpha}, A_{\beta}>0$, with the additional constraints $\alpha, \beta>0$ and $\alpha+\beta>2$. Then we have

$$
\begin{aligned}
\langle Y\rangle_{\mathrm{E}} & =\frac{1}{P(\mathrm{E})} \sum_{k=1}^{\infty} k^{1-\alpha-\beta}\left(k^{\alpha} P\left(\mathrm{E} \mid \mathrm{Y}_{\mathrm{k}}\right)\right)\left(k^{\beta} P\left(\mathrm{Y}_{\mathrm{k}}\right)\right) \\
& \leq \frac{1}{P(\mathrm{E})} A_{\alpha} A_{\beta} \sum_{k=1}^{\infty} k^{1-(\alpha+\beta)} \\
& <\infty
\end{aligned}
$$

because by assumption, $1-(\alpha+\beta)<-1$, ensuring the convergence of the series. In the remainder of the proof we will focus on the properties of the series

$$
\sum_{k=1}^{\infty} k P\left(\mathrm{Y}_{\mathrm{k}}\right) P\left(\mathrm{E} \mid \mathrm{Y}_{\mathrm{k}}\right)
$$

which is sufficient for examining the convergence properties of $\langle Y\rangle_{\mathrm{E}}$.

We now proceed to proving the sufficiency of the third condition. We assume that $\sum_{k=1}^{\infty} P\left(\mathrm{E} \mid \mathrm{Y}_{\mathrm{k}}\right)<\infty$ and that there is a $N_{0} \in \mathbb{N}$ such that $P\left(\mathrm{Y}_{\mathrm{k}}\right) \geq P\left(\mathrm{Y}_{\mathrm{k}+1}\right)$ for all $k \geq N_{0}$. By Dirichlet's criterion (Knopp 1964, 324), $\quad \sum_{k=1}^{\infty} k P\left(\mathrm{Y}_{\mathrm{k}}\right) P\left(\mathrm{E} \mid \mathrm{Y}_{\mathrm{k}}\right)$ converges if (i) $\sum_{k=1}^{\infty} P\left(\mathrm{E} \mid \mathrm{Y}_{\mathrm{k}}\right)<\infty$ and (ii) $k P\left(\mathrm{Y}_{\mathrm{k}}\right) \rightarrow 0$ monotonically. The first condition is fulfilled by assumption. The second clause of the criterion can, without loss of generality, be replaced by demanding that for $N_{0} \in \mathbb{R},\left(k P\left(\mathrm{Y}_{\mathrm{k}}\right)\right)_{k \in \mathbb{N}}$ be monotonically decreasing for all $k \geq N_{0}$.

Assume that the second clause of the criterion is not satisfied, and that there is a sequence of natural numbers $n_{k}$ such that

$$
n_{k} P\left(\mathrm{Y}_{\mathrm{n}_{\mathrm{k}}}\right)<n_{k+1} P\left(\mathrm{Y}_{\mathrm{n}_{\mathrm{k}+1}}\right) .
$$

Then the (sub)sequence $\left(n_{k} P\left(\mathrm{Y}_{\mathrm{n}_{\mathrm{k}}}\right)\right)_{\mathrm{k}}$ would not converge to zero, and consequently, $\left(k P\left(\mathrm{Y}_{\mathrm{k}}\right)\right)_{k}$ would not converge to zero. However, for some $k \geq N_{0}$, 
$P\left(\mathrm{Y}_{\mathrm{k}}\right)$ is by assumption a monotonically decreasing sequence. Furthermore, for such sequences, if $\sum_{k} P\left(\mathrm{Y}_{\mathrm{k}}\right)$ exists (which is the case here), then also $k P\left(\mathrm{Y}_{\mathrm{k}}\right) \rightarrow 0$ (Knopp 1964, 125). Hence, a subsequence $\left(n_{k} P\left(\mathrm{Y}_{\mathrm{n}_{\mathrm{k}}}\right)\right)_{\mathrm{k}}$ with property (13) cannot exist and the second part of the Dirichlet criterion is satisfied. Thus, the third condition of Theorem 2 is indeed sufficient.

Finally, we demonstrate the joint sufficiency of (i) $P\left(\mathrm{E} \mid \mathrm{Y}_{\mathrm{k}}\right) \rightarrow 0$ and (ii) there is an $\alpha>0$ such that

$$
\limsup _{k \rightarrow \infty} k^{2+\alpha}\left|P\left(\mathrm{E} \mid \mathrm{Y}_{\mathrm{k}}\right)-P\left(\mathrm{E} \mid \mathrm{Y}_{\mathrm{k}-1}\right)\right|<\infty .
$$

In particular, there exists a $C>0$ such that $k^{2+\alpha}\left|P\left(\mathrm{E} \mid \mathrm{Y}_{\mathrm{k}}\right)-P\left(\mathrm{E} \mid \mathrm{Y}_{\mathrm{k}-1}\right)\right| \leq C$. Moreover, let $C^{\prime}:=2 C \sum_{k=1}^{\infty} 1 / k^{1+\alpha}$.

By Abel's formula (Knopp 1964, 322), we can rewrite the partial sums of the series $\sum_{k=1}^{\infty} k P\left(\mathrm{Y}_{\mathrm{k}}\right) P\left(\mathrm{E} \mid \mathrm{Y}_{\mathrm{k}}\right)$ in the following way:

$$
\begin{aligned}
\sum_{k=1}^{N} k P\left(\mathrm{Y}_{\mathrm{k}}\right) P\left(\mathrm{E} \mid \mathrm{Y}_{\mathrm{k}}\right)= & \sum_{k=1}^{N}\left(\sum_{j=1}^{k} j P\left(\mathrm{Y}_{\mathrm{j}}\right)\right)\left(P\left(\mathrm{E} \mid \mathrm{Y}_{\mathrm{k}}\right)-P\left(\mathrm{E} \mid \mathrm{Y}_{\mathrm{k}+1}\right)\right) \\
& +\left(\sum_{j=1}^{N} j P\left(\mathrm{Y}_{\mathrm{j}}\right)\right) P\left(\mathrm{E} \mid \mathrm{Y}_{\mathrm{N}+1}\right) .
\end{aligned}
$$

Note that the re-ordering of the terms does not affect the convergence properties since (12) has only positive members. It is now sufficient to show that both summands on the right side are uniformly bounded in $N$ since this would mean that (12) has bounded partial sums and is thus convergent.

We begin by showing that the first summand is uniformly bounded:

$$
\begin{aligned}
& \left|\sum_{k=1}^{N}\left(\sum_{j=1}^{k} j P\left(\mathrm{Y}_{\mathrm{j}}\right)\right)\left(P\left(\mathrm{E} \mid \mathrm{Y}_{\mathrm{k}}\right)-P\left(\mathrm{E} \mid \mathrm{Y}_{\mathrm{k}+1}\right)\right)\right| \\
\leq & \sum_{k=1}^{N}\left(\sum_{j=1}^{k} \frac{j}{k} P\left(\mathrm{Y}_{\mathrm{j}}\right)\right) \frac{1}{k^{1+\alpha}} k^{2+\alpha}\left|P\left(\mathrm{E} \mid \mathrm{Y}_{\mathrm{k}}\right)-P\left(\mathrm{E} \mid \mathrm{Y}_{\mathrm{k}+1}\right)\right| \\
\leq & C \sum_{k=1}^{N}\left(\sum_{j=1}^{k} P\left(\mathrm{Y}_{\mathrm{j}}\right)\right) \frac{1}{k^{1+\alpha}} \\
\leq & C \sum_{k=1}^{\infty} \frac{1}{k^{1+\alpha}} \\
\leq & C^{\prime}
\end{aligned}
$$

and the resulting bound is independent of $N$.

For the second term, because of $P\left(\mathrm{E} \mid \mathrm{Y}_{\mathrm{k}}\right) \rightarrow 0$, there is, for any $k \in \mathbb{N}$, a 
$N_{0}(k)$ such that

$$
\left(\sum_{j=1}^{k} j P\left(\mathrm{Y}_{\mathrm{j}}\right)\right) P\left(\mathrm{E} \mid \mathrm{Y}_{\mathrm{N}_{0}(\mathrm{k})}\right) \leq C^{\prime} / 2 .
$$

Then we can calculate

$$
\begin{aligned}
& \left(\sum_{j=1}^{k} j P\left(\mathrm{Y}_{\mathrm{j}}\right)\right) P\left(\mathrm{E} \mid \mathrm{Y}_{\mathrm{k}+1}\right) \\
\leq & \left(\sum_{j=1}^{k} j P\left(\mathrm{Y}_{\mathrm{j}}\right)\right)\left|P\left(\mathrm{E} \mid \mathrm{Y}_{\mathrm{k}}\right)-P\left(\mathrm{E} \mid \mathrm{Y}_{\mathrm{k}+1}\right)\right|+\left(\sum_{j=1}^{k} j P\left(\mathrm{Y}_{\mathrm{j}}\right)\right) P\left(\mathrm{E} \mid \mathrm{Y}_{\mathrm{k}+1}\right) \\
\leq & \cdots \\
\leq & \left(\sum_{j=1}^{k} j P\left(\mathrm{Y}_{\mathrm{j}}\right)\right)\left(\sum_{l=k}^{N_{0}(k)-1}\left|P\left(\mathrm{E} \mid \mathrm{Y}_{\mathrm{l}}\right)-P\left(\mathrm{E} \mid \mathrm{Y}_{\mathrm{l}+1}\right)\right|\right)+\left(\sum_{j=1}^{k} j P\left(\mathrm{Y}_{\mathrm{j}}\right)\right) P\left(\mathrm{E} \mid \mathrm{Y}_{\mathrm{N}_{0}(\mathrm{k})}\right) \\
\leq & \left(\sum_{j=1}^{k} \frac{j}{k} P\left(\mathrm{Y}_{\mathrm{j}}\right)\right)\left(\sum_{l=k}^{N_{0}(k)-1} \frac{k}{l^{2+\alpha}} l^{2+\alpha}\left|P\left(\mathrm{E} \mid \mathrm{Y}_{\mathrm{l}}\right)-P\left(\mathrm{E} \mid \mathrm{Y}_{\mathrm{l}+1}\right)\right|\right)+C^{\prime} / 2 \\
\leq & \left(\sum_{j=1}^{k} P\left(\mathrm{Y}_{\mathrm{j}}\right)\right)\left(\sum_{l=k}^{N_{0}(k)-1} \frac{C}{l^{1+\alpha}}\right)+C^{\prime} / 2 \\
\leq & C\left(\sum_{l=1}^{\infty} \frac{1}{l^{1+\alpha}}\right)+C^{\prime} / 2 \\
\leq & C^{\prime},
\end{aligned}
$$

proving the uniform boundedness of the second summand and thereby the sufficiency of the fourth and last condition for $\langle Y\rangle_{E}<\infty$.

Proof of Proposition 2: By a straightforward application of Bayes' Theorem:

$$
\begin{aligned}
\langle Y\rangle_{\mathrm{E}} & =\sum_{k=1}^{\infty} k P\left(\mathrm{Y}_{\mathrm{k}} \mid \mathrm{E}\right)=\frac{1}{P(\mathrm{E})} \sum_{k=0}^{\infty} k P\left(\mathrm{Y}_{\mathrm{k}}\right) P\left(\mathrm{E} \mid \mathrm{Y}_{\mathrm{k}}\right) \\
& \leq \frac{1}{P(\mathrm{E})} \sum_{k=0}^{\infty} k P\left(\mathrm{Y}_{\mathrm{k}}\right)=\frac{1}{P(\mathrm{E})}\langle Y\rangle \\
& <\infty
\end{aligned}
$$

\section{B Proof of Theorem 3 in Section 4}

$\mathrm{F}_{\mathrm{A}}$ confirms $\mathrm{T}$ if and only if $P\left(\mathrm{~T} \mid \mathrm{F}_{\mathrm{A}}\right)-P(\mathrm{~T})>0$, that is, if and only if

$$
\Delta:=P\left(\mathrm{~T}, \mathrm{~F}_{\mathrm{A}}\right)-P(\mathrm{~T}) P\left(\mathrm{~F}_{\mathrm{A}}\right)>0 .
$$


We now apply the theory of Bayesian Networks to the structure depicted in Figure 2, using assumption $\mathbf{A} \mathbf{1}\left(T \Perp F_{A} \mid Y\right)$ :

$$
\begin{aligned}
P\left(\mathrm{~F}_{\mathrm{A}}\right) & =\sum_{i=0}^{\infty} \sum_{j=0}^{\infty} P\left(\mathrm{~F}_{\mathrm{A}} \mid \mathrm{Y}_{\mathrm{i}}, \mathrm{D}_{\mathrm{j}}\right) P\left(\mathrm{Y}_{\mathrm{i}}, \mathrm{D}_{\mathrm{j}}\right)=\sum_{i=0}^{\infty} \sum_{j=0}^{\infty} d_{j} y_{i} f_{i j} \\
P(\mathrm{~T}) & =\sum_{k=0}^{\infty} P\left(\mathrm{~T} \mid \mathrm{Y}_{\mathrm{k}}\right) P\left(\mathrm{Y}_{\mathrm{k}}\right)=\sum_{k=0}^{\infty} t_{k} y_{k} \\
P\left(\mathrm{~T}, \mathrm{~F}_{\mathrm{A}}\right) & =\sum_{i=0}^{\infty} P\left(\mathrm{~F}_{\mathrm{A}}, \mathrm{T} \mid \mathrm{Y}_{\mathrm{i}}\right) P\left(\mathrm{Y}_{\mathrm{i}}\right)=\sum_{i=0}^{\infty} y_{i} P\left(\mathrm{~F}_{\mathrm{A}} \mid \mathrm{Y}_{\mathrm{i}}\right) P\left(\mathrm{~T} \mid \mathrm{Y}_{\mathrm{i}}\right) \\
& =\sum_{i=0}^{\infty} y_{i} t_{i}\left(\sum_{j=0}^{\infty} P\left(\mathrm{~F}_{\mathrm{A}} \mid \mathrm{Y}_{\mathrm{i}}, \mathrm{D}_{\mathrm{j}}\right) P\left(\mathrm{D}_{\mathrm{j}} \mid \mathrm{Y}_{\mathrm{i}}\right)\right) \\
& =\sum_{i=0}^{\infty} \sum_{j=0}^{\infty} d_{j} y_{i} t_{i} f_{i j}
\end{aligned}
$$

Hence, we obtain, using $\sum_{k \in \mathbb{N}} y_{k}=1$,

$$
\begin{aligned}
\Delta & =\left(\sum_{i=0}^{\infty} \sum_{j=0}^{\infty} d_{j} y_{i} t_{i} f_{i j}\right)-\left(\sum_{i=0}^{\infty} \sum_{j=0}^{\infty} d_{j} y_{i} f_{i j}\right)\left(\sum_{k=0}^{\infty} y_{k} t_{k}\right) \\
& =\left(\sum_{i=0}^{\infty} \sum_{j=0}^{\infty} d_{j} y_{i} t_{i} f_{i j}\right)\left(\sum_{k=0}^{\infty} y_{k}\right)-\left(\sum_{i=0}^{\infty} \sum_{j=0}^{\infty} d_{j} y_{i} f_{i j}\right)\left(\sum_{j=0}^{\infty} t_{k} y_{k}\right) \\
& =\sum_{i=0}^{\infty} \sum_{j=0}^{\infty} \sum_{k=0}^{\infty}\left(d_{j} y_{i} y_{k} t_{i} f_{i j}-d_{j} y_{i} y_{k} t_{k} f_{i j}\right) \\
& =\sum_{j=0}^{\infty} d_{j} \sum_{i=0}^{\infty} \sum_{k \neq i=0}^{\infty} y_{i} y_{k} f_{i j}\left(t_{i}-t_{k}\right) \\
& =\sum_{j=0}^{\infty} d_{j} \sum_{i=0}^{\infty} \sum_{k>i}\left(y_{i} y_{k} f_{i j}\left(t_{i}-t_{k}\right)+y_{k} y_{i} f_{k j}\left(t_{k}-t_{i}\right)\right) \\
& =\sum_{j=0}^{\infty} d_{j} \sum_{i=0}^{\infty} \frac{1}{2} \sum_{k \neq i=0}^{\infty} y_{i} y_{k}\left(f_{i j}\left(t_{i}-t_{k}\right)+f_{k j}\left(t_{k}-t_{i}\right)\right) \\
& =\frac{1}{2} \sum_{i=0}^{\infty} \sum_{j=0}^{\infty} \sum_{k \neq i=0}^{\infty} d_{j} y_{i} y_{k}\left(t_{i}-t_{k}\right)\left(f_{i j}-f_{k j}\right) \\
& >0
\end{aligned}
$$

because of A2-A5 taken together.

\section{References}

Bovens, L., and S. Hartmann (2003): Bayesian Epistemology. Oxford: Oxford University Press. 
Carlin, B., and T. Louis (2000): Bayes and Empirical Bayes Methods for Data Analysis. London: Chapman \& Hall.

Cushing, J. (1994): Quantum Mechanics: Historical Contingency and the Copenhagen Hegemony. Chicago: The University of Chicago Press.

Dawid, R. (2006): Underdetermination and Theory Succession from the Perspective of String Theory. Philosophy of Science 73: 298-322.

Dawid, R. (2009): On the Conflicting Assessments of the Current Status of String Theory. Philosophy of Science 76: 984-996.

Douven, I. (2011): Abduction, in: E. Zalta (ed.), The Stanford Encyclopedia of Philosophy (Spring 2011 Edition).

Fraassen, B. van (1980): The Scientific Image. Oxford: Oxford University Press.

Hahn, U., and M. Oaksford (2007): The Rationality of Informal Argumentation: A Bayesian Approach to Reasoning Fallacies. Psychological Review 114: 704-732.

Hájek, A., and S. Hartmann (2010): Bayesian Epistemology, in: J. Dancy et al. (eds.), A Companion to Epistemology. Oxford: Blackwell, 93-106.

Hartmann, S., and J. Sprenger (2010): Bayesian Epistemology, in: S. Bernecker and D. Pritchard (eds.), Routledge Companion to Epistemology. London: Routledge, 609-620.

Hitchcock, C., and E. Sober (2004): Prediction Versus Accommodation and the Risk of Overfitting. British Journal for the Philosophy of Science 55: $1-34$.

Howson, C., and P. Urbach (2006): Scientific Reasoning: The Bayesian Approach. Third Edition. La Salle: Open Court.

Kahn, J., S. Landsburg, and A. Stockman (1992): On Novel Confirmation. British Journal for the Philosophy of Science 43: 503-516.

Knopp, K. (1964): Theorie und Anwendung der unendlichen Reihen. Berlin: Springer.

Lipton, P. (2004): Inference to the Best Explanation. London: Routledge. 
Sober, E. (2009): Absence of Evidence and Evidence of Absence: Evidential Transitivity in Connection with Fossils, Fishing, Fine Tuning and Firing Squads. Philosophical Studies 143: 63-90.

Stanford, K. (2006): Exceeding Our Grasp: Science, History, and the Problem of Unconceived Alternatives. New York: Oxford University Press.

Walton, D. (1995): Arguments from Ignorance. Philadelphia: Penn State University Press. 